\title{
Tra essere e agire I mille volti della paura nella trilogia di Batman di Christopher Nolan
}

\author{
di Lorenza Di Francesco \\ Università degli Studi di Modena e Reggio Emilia \\ lorenzadifrancesco@ymail.com
}

\begin{abstract}
The aim of this study is a semiotic analysis of the Nolan's Batman series (Batman Begins/2005, The Dark Knight/2008, The Dark Knight Rises/2012). It goes over dimension of figural, space and passion in order to highlight fear as a complex, primitive and moving passion that guides characters transformation, actions and narrative construction of these three movies.
\end{abstract}

\section{Parole chiave}

Cinema, semiotics, visual culture, visual semiotics

\section{Sommario}

La paura figurale

La paura come soggetto manipolatore

L'opposizione paura/vergogna al centro della narrazione

Le sfumature della paura e l'ordinamento spaziale

Conclusioni

Bibliografia 
Se l'innamorata di sempre, Rachel, ammonisce Bruce Wayne riguardo al fatto che ciò che definisce un uomo è quello che fa e non quello che sente, ${ }^{1}$ valorizza cioè un agire rispetto a un sentire, in questo studio mostreremo come, nonostante tutta l'azione - gli inseguimenti, i voli, le lotte - i tre Batman (Batman Begins/2005, The Dark Knight/2008, The Dark Knight Rises/2012) di Christopher Nolan facciano perno attorno alla paura. Stato d'animo viscerale e primario che articola lo svolgimento narrativo dei tre film muovendosi lungo le direttrici della figuralità, dello spazio e della passione e che, grazie a questa complessa costruzione, trasforma un bambino terrorizzato prima in un ragazzo che usa l'inquietudine degli altri poi in un uomo che affranca se stesso grazie alla propria paura. Tutto all'ombra della città di Gotham, oscuro ma malcelato Destinante (v. Greimas, Courtés 1979) dell'intera narrazione.

\section{La paura figurale}

La figura del pipistrello in Batman è sicuramente una figura di rilievo. Essa è lessicalizzata nel nome dell'eroe e ravvisabile figurativamente nel suo sembiante; pipistrelli aleggiano in tutti e tre i film e l'intera atmosfera, attiva nel produrre effetti di senso emotivi e passionali, ha più a che fare con l'animale notturno che con la brillante vita che ci immagineremmo per un rampollo milionario. Anche quando si parla del sentimento della paura in Batman si pensa subito alla figura del pipistrello, ma a una riflessione attenta non è questa a scatenare la paura del piccolo Bruce e, in seguito, a ispirare in Bruce adulto il travestimento dell'eroe di Gotham. Infatti, caduto in un pozzo nei pressi di casa, il bambino non è impressionato dallo stuolo di animali che gli volano sopra la testa ma dal fatto che, di quella "nuvola nera" in movimento vorticoso e ascendente, lui non riesca a distinguere nulla. Non riconosce in essa nessun elemento del mondo che conosce, che gli è familiare e che ama. Nei Batman di Nolan perciò la paura non deriva dalla visione di qualcosa bensì dalla sua non-visione. Basta osservare le inquadrature dei pipistrelli per accorgersi di come qui la paura sia legata più a una situazione di cecità e di ignoranza in chi vede che non a una situazione di oggettivo pericolo esterno: si tratta, nella maggior parte dei casi, di riprese di questi animali in rapido volo, e questo celere e, a volte, scomposto movimento impedisce all'occhio di distinguere i particolari fisici delle bestie (fig. 1). ${ }^{2}$

Allo stesso modo, una volta divenuto adulto, Bruce recupera questa paura ancestrale per dirigerla da sé ai malfattori di Gotham. Egli crea un eroe, lo porta alla ribalta della città e della cronaca riservandogli allo stesso tempo un alone di profondo mistero. Come fa tutto ciò? In due semplici modi. Dipingendo

1 It's not who you are underneath, it's what you do that defines you.

2 Nella scena citata, come in tutti e tre i film, lo spettatore non vede mai distintamente i pipistrelli. L'unica esperienza mediale (Eugeni 2010) consentita è avvertire sensorialmente - oltre a vedere l'immagine si sente un fastidioso rumore che delle "cose" nere si stanno muovendo (spesso attraversando l'inquadratura da una parte all'altra). Lo spettatore riconosce dei pipistrelli solo grazie a un sapere sociale (Eugeni 1999) relativo al mondo degli animali e a un sapere culturalmente più specifico relativo alla storia di Batman. 


\section{Oculatiux_saggi}

Tra essere e agire | Lorenza Di Francesco

di nero una sorta di moderna armatura e mezzo arsenale bellico dell'azienda paterna, e agendo di notte, ossia all'oscuro (fig. 2). Possiamo perciò affermare che Batman è una maschera che delinea una paura senza però renderla chiaramente riconoscibile. Si tratta di una paura che viene avvertita, sentita fin dentro le ossa, ma che allo stesso tempo non è nominabile perché non possiede una controparte nel mondo reale, ed è forse questa la sua forza.

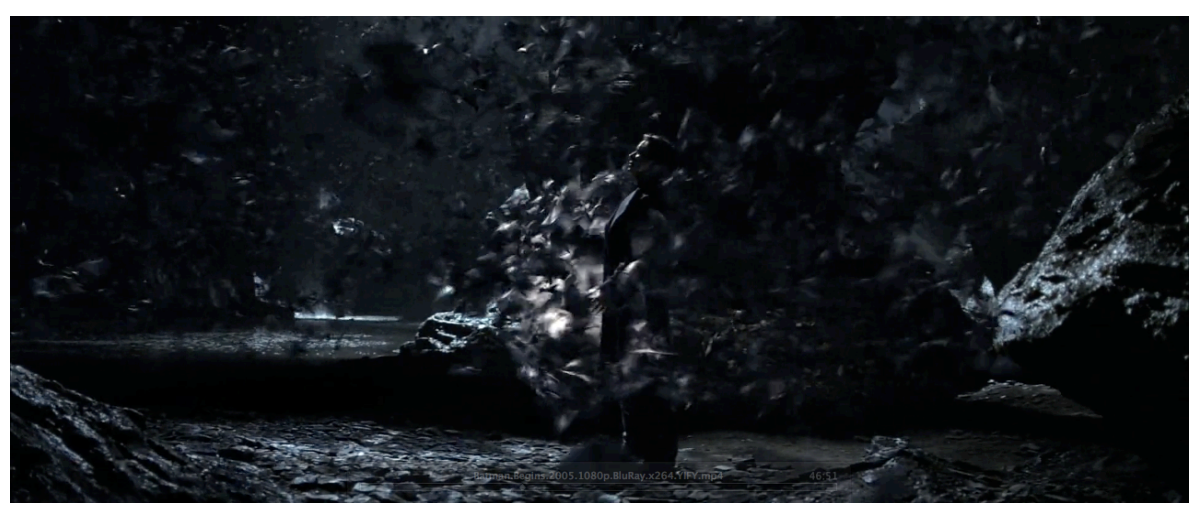

Figura 1.

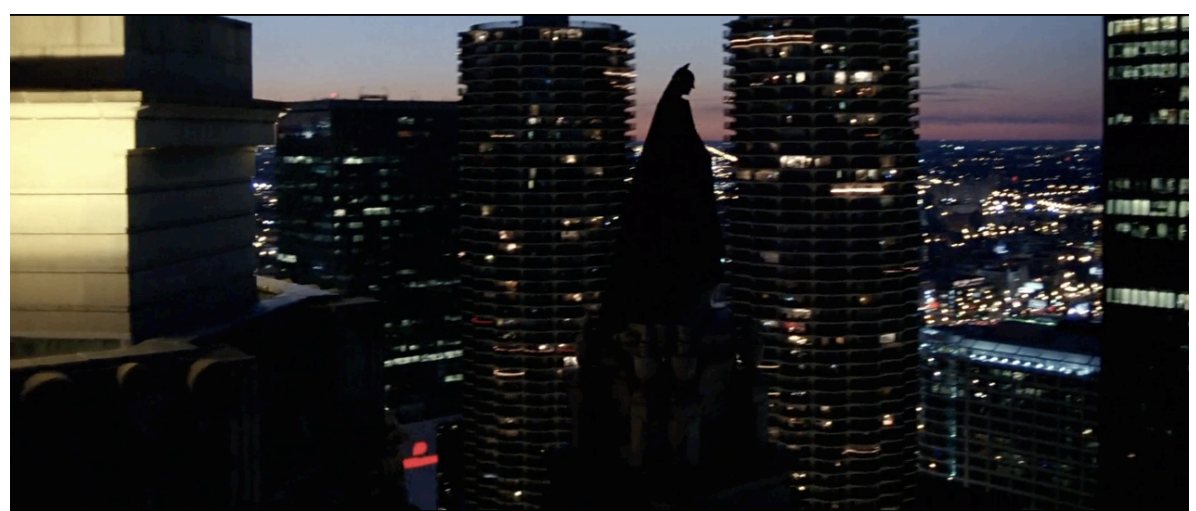

Figura 2.

\section{La paura come soggetto manipolatore}

La paura, fear, nei tre film di Batman non è solo quella che si vede, o intravede come abbiamo detto. Essa è spesso presente anche nei dialoghi del film, ${ }^{3}$ quindi in qualità di soggetto o oggetto degli enunciati. Ed è in questa dimensione che la paura viene descritta nei termini di agente manipolatore (v. Greimas, Courtés 1979), ossia di elemento che, diretto e ri-diretto da un attore all'altro, interviene nei rapporti tra i protagonisti strutturandone $\mathrm{i}$

3 Esemplare, in Batman Begins, è una delle prime battute del film che presenta allo spettatore il personaggio di Bruce Wayne, erede della più ricca famiglia della metropoli di Gotham, what do you fear, Bruce?, "di cosa hai paura, Bruce?" pronunciata da una voce fuori campo sul primo piano del giovane uomo. Ma riferimenti alla paura sono disseminati lungo tutto il film. 
processi d'azione. La paura infatti, pur immobilizzando alcuni attori nel loro stesso terrore, ${ }^{4}$ è un sentimento che si muove all'interno della narrazione segnando il destino dell'intera città. Essa rende traumatica l'infanzia di Bruce Wayne (la già ricordata disavventura del pozzo), determina l'omicidio dei suoi genitori (la paura di perdere i propri cari fa compiere una mossa azzardata e fatale al padre del bambino), motiva l'invenzione dell' "uomo pipistrello" e infine scioglie lo stesso Bruce da quella che potremmo definire la sua prigionia passionale. In particolare, questa liberazione finale si realizza perché nell'intimo del protagonista agisce il dualismo paura/vergogna.

\section{L'opposizione paura/vergogna al centro della narrazione}

I Batman di Nolan costruiscono o ricostruiscono - se si pensa ai tre film come traduzioni intersemiotiche (v. Dusi 2006) dal fumetto originale ${ }^{5}-$ un mondo fantastico che segue tuttavia molte norme di quello reale, prima fra tutte l'organizzazione della collettività attorno alle categorie della paura e della vergogna. Ma se nel mondo reale "la presenza di entrambe e la loro differenziazione sono evidentemente indispensabili al meccanismo della cultura" (Lotman, Uspenskij 1975: 272), a Gotham tutto è regolato invece attorno alla sola paura. Pensiamo ai dispositivi, coercitivi e non, che nei film hanno come obiettivo contrastare la mafia e la delinquenza: le molteplici leggi e ordinamenti giudiziari, le fondazioni benefiche, il treno-metropolitana che attraversa in poco tempo la città. In fin dei conti, lo stesso Batman, nel secondo e terzo capitolo della saga, viene valorizzato come cavaliere oscuro Dark Knight - ossia come difensore misterioso dell'ordinamento di Gotham. La vergogna agisce solo all'interno di Bruce, dilaniato dai sensi di colpa per la morte dei genitori. Ed è da questo opposizione che prende il via tutta la vicenda che conosciamo.

Il primo sentimento esperito dal bambino è infatti senz'altro la paura ma, immediatamente dopo aver subìto lo shock dei pipistrelli, Bruce prova anche la vergogna, una vergogna però sempre legata alla paura. Il bambino si vergogna, davanti al padre, suo modello di vita, di avere provato paura, di essere un codardo ${ }^{6}$. Sin dalle prime scene di Batman Begins, quindi, il mondo pas-

4 In Batman Begins, ad esempio, il dottor Crane/Spaventapasseri è un "cattivo" che somministra ai malcapitati un gas che materializza le paure più profonde di chi lo inala, causando spesso una vera e propria paralisi. È interessante notare come, grazie a questo gas, la paura sia raffigurata in tutti i suoi minimi particolari: sia quindi resa iconica. Si verifica perciò un processo inverso rispetto a quello riscontrato generalmente nella trilogia.

5 Il primo fumetto di Batman è edito in America dalla DC Comics nella primavera del 1940, quindi le tante trasposizioni cinematografiche sono da intendersi come traduzioni della medesima narrazione da un medium all'altro. Per approfondimenti v. Dusi, N., Il cinema come traduzione. Da un medium all'altro: letteratura, cinema, pittura, Utet, Torino 2006.

6 Da notare come in Batman la paura non è mai legata allo spazio, per cui nonostante spesso i luoghi della narrazione e dell'azione coincidano con ambienti angusti, bui e sconosciuti - il pozzo della casa paterna, la prigione verticale di Bane, le fogne di Gotham - gli attori, e in particolare il protagonista principale, sembrano non avvertire mai la pericolosità che normalmente verrebbe associata a questi luoghi. 
sionale del protagonista si costruisce attorno a una tensione, quella tra vergogna e paura. È il fatto che l'una dipenda dall'altra a innescare questa tensione - la vergogna ha per oggetto la paura - e sono le modulazioni di questa tensione a determinare gli sviluppi narrativi dei tre film. Così se lo spettatore assiste, sempre sul piano della narrazione, a un tentativo costante di accantonare, celare il tema della vergogna, quello della la paura viene al contrario continuamente portato alla ribalta e quasi dissezionato nelle sue più intime parti. Pensiamo al fatto che solo in momenti puntuali dei tre film si accenna alla vergogna del protagonista, 7 mentre la paura, da passione esperita sul proprio corpo, diventa dapprima istinto da dominare, dopo obnubilamento indotto negli altri.

\section{Le sfumature della paura e l'ordinamento spaziale}

La paura nella trilogia di Nolan, di cui stiamo provando ad analizzare parte della ricchezza e potenzialità, vale perciò non come semplice valore ma come configurazione passionale (questa è la nostra tesi). $\mathrm{E}$ in quanto patema complesso, grazie alle sue sfumature, opera una spazializzazione all'interno del discorso filmico. I tre film infatti presentano lo spazio cittadino di Gotham organizzato secondo un "sopra" e un "sotto" per cui le scene si svolgono o sulla terraferma o nel sottosuolo. Sappiamo, a tal proposito, che e peripezie di Batman si compiono tra o dentro i grattacieli ma che il suo armamentario è conservato nei sotterranei della sua villa, addirittura al di sotto di un fiume. La paura, in questo caso, rafforza la presenza di questo asse orizzontale ripartendo i "buoni" dai "cattivi" o, meglio, gli integrati dagli emarginati della città. Se è vero infatti che essa è un sentimento condiviso da tutti nel film, è una passione collettiva come vedremo tra poco, è anche vero che esiste una paura di chi vive $s u$ Gotham e una di chi vive sotto di essa. La prima identifica i benestanti, chi possiede proprietà e affetti e teme per l'incolumità di questi ultimi. È la paura del piccolo Bruce verso i pipistrelli come quella di Bruce adulto che, cacciato dal college, viaggia per il mondo alla ricerca prima dei criminali poi dei miserabili. Si tratta della paura dell'ignoto, di ciò che non si conosce, spesso perché non si vede o non si riesce a delineare. È il sentimento che segna il distacco dal mondo: in una parola, l'ignoranza. La seconda appartiene a chi non ha nulla, nessun bene, nessun amore o amico e teme di perdere anche la speranza. È la paura del miserabile Chill che rapina e uccide i genitori di Bruce per pochi soldi come quella di Bane, figlio di detenuti e perciò nato e cresciuto in una prigione sotterranea. È il sentimento che segna il distacco dalla vita: è la disperazione.

Nei primi due Batman - Batman Begins e The Dark Knight - la paura è sostanzialmente un sentimento che si fronteggia, si domina e si usa come un'arma micidiale ma non è un sentimento che si prova, che si esperisce fino in fondo. In The Dark Knight Rises, l'ultimo film della trilogia, invece, la

\footnotetext{
7 Di regola negli scambi verbali con il maggiordomo Alfred, una sorte di "coscienza"
} di Bruce. 
paura non viene solo descritta e rappresentata nella sua valenza cognitiva e pragmatica ma vale sopratutto come esperienza estesica e passionale. Lo spettatore scopre così, seguendo la vicenda del protagonista, che è solo entrando in contatto con la propria inquietudine che si raggiunge la libertà, l'affrancamento dalla prigionia fisica e da quella mentale. Facendosi carico della propria paura di morire, Bruce riesce a spiccare il salto che lo porta fuori dalla prigione-fossa, e accettando il personale senso di colpa in relazione alla morte dei genitori, riesce a costruire e dare un senso alla sua vita (una delle ultime significative inquadrature di The Dark Knight Rises ha al centro Bruce con la sua famiglia).

Ma la paura, nella trilogia di Batman, rappresenta anche un "sentire" proprio di tutta Gotham - e ciò rende ancora più stretto il legame tra la città e l'eroe, legame valorizzato in diverse immagini create ad hoc per i trailers (fig. 3). Specificatamente, la paura qui come passione collettiva (cfr. Del Marco, Pezzini, a cura di, 2012) appare come un sentimento che immobilizza, rende catatonica la città, e pertanto essa è una pulsione sfruttata appieno dai "cattivi" della narrazione. Pensiamo al diabolico piano di Bane, in The Dark Knight Rises, che prevede una sorta di suicidio di massa autoindotto dagli abitanti, immobilizzati tra il terrore di una morte imminente (nel sottosuolo è stato piazzato un gigantesco ordigno) e la fiducia mal riposta in Bane, che promette loro una possibilità di salvezza. Unica eccezione a questo status quo è portata in scena grazie alla figura di Joker, l'antieroe di The Dark Knight, che introduce il caos; elemento che, per sua stessa natura, rompe l'ordine instaurato dalla paura. Attraverso il caos - Joker non segue nessun piano preordinato e agisce simile a una scheggia impazzita - la paura come dimensione durativa di Gotham viene messa in crisi. Allo stesso modo in cui Batman utilizza il sentimento della paura per innervare le proprie azioni, quelle dei nemici e scatenare le reazioni nei cittadini, Joker cerca di conquistare la città liberandola dai percorsi morali obbligati e dalla più stretta logica. Ma il suo tentativo, rimanendo circoscritto al secondo film della trilogia, rappresenta più un intermezzo passionale tra Batman Begins e The Dark Knight Rises, che uno sviluppo decisivo per l'intera narrazione. ${ }^{8}$

\section{Conclusioni}

Il sentimento della paura in Batman modula la dimensione del principale protagonista, trasformandolo via via da soggetto passivo - che salvaguarda il proprio percorso di vita e la propria incolumità - a soggetto attivo - che sacrifica se stesso per la comunità - e infine a soggetto senziente - che si mette in contatto con i propri sentimenti e il proprio corpo per raggiungere la libertà non solo fisica ma anche psicologica. Ciò avviene perché, situandosi nel

8 Anche la riflessione di Slavoj Zižek, incentrata su una lettura politica della trilogia e sulla circolazione in essa dei valori della legge, dell'ordine, dell'amore e della rivoluzione, concorda sulla marginale rilevanza della figura di Joker, ora ridotto a un "apparente avversario", ora uniformato a uno dei "gangsters pazzi”. S. Zižek, S., "The Politics of Batman", in New Statesman, London, 23 August 2012. 


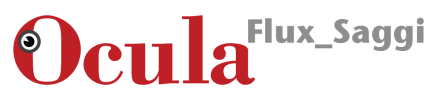

Tra essere e agire | Lorenza Di Francesco

cuore valoriale della narrazione, la paura vale come complessa configurazione passionale che regola l'ottusità e la sensibilità emotiva degli attori, il loro adattamento e la loro reattività agli eventi, addirittura l'introduzione e l'espulsione di nuovi valori, come il caos portato da Joker. Elemento di forte disturbo quest'ultimo, ma messo in gioco solo per sparigliare per un breve lasso di tempo l'ordine della narrazione e prontamente espulso per ripristinare in maniera ancora più salda la disciplina della paura. 


\section{Ocula $a^{\text {Flux__aggi }}$}

Tra essere e agire | Lorenza Di Francesco

\section{Bibliografia}

Del Marco, V., Pezzini I. (a cura di), Passioni collettive. Cultura, politica e società, La Nuova Cultura, Roma 2012.

Dusi, N., Il cinema come traduzione. Da un medium all'altro: letteratura, cinema, pittura, Utet, Torino 2006.

Eugeni, R., Film, sapere, società. Per un'analisi sociosemiotica del testo cinematografico, Vita e Pensiero, Milano 1999.

- , Semiotica dei media. Le forme dell'esperienza, Carocci, Roma 2010.

Greimas, A. J., Courtés, J., Sémiotique. Dictionnaire raisonné de la théorie du langage, Hachette, Paris 1979; trad. it. Fabbri, P. (a cura di), Semiotica. Dizionario ragionato della teoria del linguaggio, Mondadori, Milano 2007.

Lotman, J., M., Uspenskij, B., A., Stat’i po tipologii kul'tury. Materialy k kursu teorii literatury, Tartu, 1970; trad. it., Tipologia della cultura, Bompiani, Milano 1975.

Zižek, S., "The Politics of Batman", in New Statesman, London 23 August 2012; trad. it. "Repubblica popolare di Gotham", in Internazionale n. 965, 7/13 settembre 2012. 